\title{
Some Hints for the Design of Digital Chaos-Based Cryptosystems: Lessons Learned from Cryptanalysis
}

\author{
David Arroyo*, Gonzalo Alvarez* and Shujun Li** \\ * Instituto de Física Aplicada, Consejo Superior de Investigaciones \\ Cientificas, Serrano 144, 28006 Madrid, Spain (e-mail: \\ david.arroyo@iec.csic.es). \\ ** Fachbereich Informatik und Informationswissenschaft, Universität \\ Konstanz, Fach M697, Universitätsstraße 10, 78457 Konstanz, \\ Germany.
}

\begin{abstract}
In this work we comment some conclusions derived from the analysis of recent proposals in the field of chaos-based cryptography. These observations remark a number of major problems detected in some of those schemes under examination. Therefore, this paper is a list of what to avoid and to pay special attention to when considering chaos as source of new strategies to conceal and protect information.
\end{abstract}

Keywords: Chaos, cryptography, cryptanalysis

\section{INTRODUCTION}

The core of digital chaos-based cryptography is the selection of a good chaotic map for a given encryption scheme. Actually, the presence of chaos does not guarantee the security of an encryption algorithm (Kocarev, 2001). A good digital cryptosystem based on chaos should not be just the concomitance of a chaotic map and an encryption 'architecture, but the result of their synergical association. Indeed, the quality of a chaotic map for cryptography must be evaluated not just with considerations on its dynamic properties, but also with considerations on the needs of the sustaining encryption architecture. In other words, from a general point of view it is not possible to design ' chaotic cryptosystems satisfying the chaotic-system-free property (Li, 2003, p. 30) and, as a result, the selection of a certain encryption scheme demands the selection of a group of chaotic maps satisfying a certain set of dynamical properties. Finally, digital chaos-based cryptography is implemented on computers and thus the problem derived from finite-precision computation must be evaluated and conveniently handled during the design stage. This work illustrates the problems with three elements involved in the design of digital chaos-based cryptosystems, i.e., the selection of a chaotic map (Sec. 2), the selection of an encryption architecture (Sec. 3) and the implementation of the encryption system (Sec. 4).

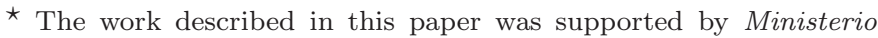
de Educación y Ciencia of Spain, research grant SEG2004-02418, CDTI, Ministerio de Industria, Turismo y Comercio of Spain in collaboration with Telefónica I+D, Project SEGUR@ with reference CENIT-2007 2004, CDTI, Ministerio de Industria, Turismo y Comercio of Spain in collaboration with SAP, project HESPERIA (CENIT 2006-2009), and Ministerio de Ciencia e Innovación of Spain in collaboration, project CUCO (MTM2008-02194). Shujun $\mathrm{Li}$ was supported by a fellowship from the Zukunftskolleg of the Universität Konstanz, Germany.
}

\section{PROBLEMS WITH THE SELECTION OF THE CHAOTIC SYSTEM}

Problem 1. Definition of the key leading to nonchaotic behavior. In some chaos-based cryptosystems the control parameters of the underlying chaotic systems are determined by the secret key. If the link between the secret key and the control parameters is not established carefully, then it is possible that the underlying chaotic system evolves in an non-chaotic way, which further erodes the confusion and diffusion properties required by the resulting cryptosystem.

The chaotic systems used as base of cryptosystems are defined in a parametric way such that their dynamics depends on one or several control parameters. Moreover, those chaotic systems are dynamical systems which show a chaotic behavior for certain values of the associated control parameter(s). Therefore, the design of a cryptosystem based on any of those dynamical systems must be done by guaranteeing the use of the set of values for the control parameter(s) leading to chaos. Otherwise, the underlying dynamical system associated to the cryptosystem (or encryption system) evolves non-chaotically, which implies the reduction of the level of entropy in the ciphertext (i.e., the output of the cryptosystem) and of the influence on the ciphertext of a change in the plaintext (i.e., the input of the cryptosystem). This problem is specially relevant when the design of the cryptosystem is based on a dynamical system with chaotic behavior only for a set of disjoint intervals of values of the control parameter(s). This is the case of the logistic map and the Hénon map, which have been used in (Pareek et al., 2003) and in (Chee and Xu, 2006) respectively without a thoroughly analysis of their dynamics (Alvarez et al., 2003a; Arroyo et al., 2008b). As a conclusion, it is highly advisable to use dynamical systems with chaotic behavior for all the values of the control 
parameter(s). That is, robust chaotic systems (Banerjee et al., 1998) should be used instead of nonrobust ones.

Problem 2. Nonuniform probability distribution function. In some chaos-based encryption architectures the confusion and/or diffusion properties depends on the probability distribution function of the orbits derived from the selected chaotic systems. If that distribution is not uniform and independent of the value(s) of control parameter(s), then the quality of the diffusion process is reduced.

The iteration of a chaotic map can be used to generate pseudo-random sequences to encrypt the plaintext. The encryption procedure could be performed by different ways, but all of them demand the equiprobability of all the states contained in the pseudo-random sequences. If this requirement is not satisfied, then the conditional entropy of the ciphertext with respect to the plaintext may be large enough to leak information about relationships between the output and the input of the target cryptosystem (see the entropy attack in (Alvarez et al., 2003c)). This effect is specially significant for image encryption, as pointed out recently by Li et al. (2007) (see Fig. 5 of their paper). As a remedy, chaotic maps with a uniform probability distribution function should be selected as base of this kind of cryptosystems, being the family of piecewise linear chaotic maps (Li et al., 2005) a good option.

Problem 3. Return map reconstruction. The ciphertext of some cryptosystems make it possible to reconstruct a return map of the underlying chaotic system. If such a return map is meaningful, then an attacker may be able to infer the value(s) of the control parameter(s) that govern the evolution of the chaotic system.

The most direct way to estimate the control parameter(s) from a chaotic orbit is to plot $x_{n+1}$ versus $x_{n}$, which is actually the chaotic map itself. If this representation shows a simple function between $x_{n+1}$ and $x_{n}$, then it could be possible to infer the control parameter. In (Skrobek, 2008) a chosen-ciphertext attack is used to build a discretized version of the logistic map which further leads to the estimation of the control parameter. One solution against this kind of attack is to shuffle/truncate the chaotic orbit before using it for encryption, which randomizes the plot of the the return map.

\section{PROBLEMS WITH THE ENCRYPTION ARCHITECTURE}

Problem 4. Bad definition of the ciphertexts. A bad definition of the ciphertext derived from a chaos-based cryptosystem could allow the estimation of the initial condition(s) and/or the control parameter(s) of the underlying chaotic system. This problem is present in some chaos-based cryptosystems whose ciphertext is given by fragments of orbits, sampled versions of the orbits, or discretized versions of the orbits of the underlying chaotic systems.

A $N$-dimensional discrete-time chaotic map is defined by the rule of evolution

$$
\mathbf{x}_{n+1}=f_{\boldsymbol{\lambda}}\left(\mathbf{x}_{n}\right),
$$

and, as a result, the ciphertext can not be the orbits of the map since it may allow the estimation of $\boldsymbol{\lambda}$ from $N+1$ or a bit more consecutive units of ciphertext (see (Arroyo et al., 2008c)). If the invariant set of the chaotic map has a size dependent on the control parameter(s), even sampled versions of the orbits may allow the estimation of the control parameter(s). This is the case of the cryptosystems reported in (García and Jiménez, 2002; Pisarchik et al., 2006) and cryptanalyzed in (Alvarez et al., 2003b; Arroyo et al., 2008d). Finally, the theory of symbolic dynamics can be used when the ciphertext allows to get the symbolic sequences of the orbits of a chaotic map (see (Alvarez et al., 2003a; Arroyo et al., 2008a)).

Problem 5. Efficiency of the cryptosystem depending on the value of the key. If the encryption and decryption times depend on the key or a sub-key, then a timing-attack can be performed to estimate the (sub-)key.

Some encryption architectures perform the transformation of the plaintext into the ciphertext through several encryption rounds. Additionally, in each encryption round a chaotic map is iterated $n$ times. Since the encryption and decryption time has to be constant and independent of the value of the key, it is not a good practice to select the number of encryption rounds and $n$ as part of the key. Otherwise, a timing-attack based on the analysis of the encryption and decryption time can be used for the partial estimation of the secret key (see (Arroyo et al., 2008d)), which is a serious security flaw. Instead, the number of encryption rounds and the number of iterations of the map should be public parameters of the cryptosystem.

Problem 6. Faulty derivation of the parameters of the chaotic system from the key. In some chaosbased cryptosystems the key is used to derive the values of the parameters necessary to iterate a chaotic system and finally encrypt the information. If this mapping implies a reduction of the key space, i.e., that it is only used a subset of the possible values of those parameters, then a bruteforce attack on the values of the parameter could be much less demanding than the one on the secret key.

One important step in the design of a chaos-based cryptosystem is to decide what the key is. One possibility is to use the control parameter(s) and the initial condition(s) of the underlying chaotic system(s) as the secret key or as part of the secret key. Another option is to establish the values of the control parameter(s) and the initial condition(s) of the map(s) from the secret key through a certain function. In this sense, it must be assured that the image set of that function is the whole set of possible values of the control parameter(s) and the initial condition(s). Otherwise, a brute-force attack can be performed on the reduced space of control parameter(s) and initial condition values with a lower computational cost than the one on the key space. A cryptosystem with this problem was introduced in (Pareek et al., 2003) and was later cryptanalyzed in (Alvarez et al., 2003a).

Problem 7. Encryption procedure equivalent to a mapping only dependent on the key. If the transformation of the plaintext into the ciphertext is determined by a procedure equivalent to a mapping only dependent on the key, then known/chosen-plaintext attacks may be performed to reconstruct the transformation procedure. 
In some encryption schemes the transformation of the plaintext into the ciphertext is leading either by a procedure derived using only the key, or by a sampling process on a sequence of values generated using only the key. In those situations, it could be possible to estimate either the key or to make up some function somehow equivalent to the encryption procedure. For example, if the encryption procedure consists of searching plaintexts in pseudorandom sequences generated by iterating a chaotic map, since the pseudo-random sequence remains unchanged unless the key is modified, then it is possible to reconstruct the pseudo-random sequence through a chosen-plaintext attack (see (Alvarez et al., 2004a,b)). This problem also exists in those schemes where the encryption procedure consists of a permutation-only stage which is fixed unless the control parameter(s) and initial condition(s) change, i.e., unless the the secret key is updated (see ( $\mathrm{Li}$ et al., 2008 b) for a general qualitative analysis of this attack). As a conclusion, the encryption function that transforms a unit of plaintext into a unit of ciphertext should depend on the key and on the whole plaintext.

\section{IMPLEMENTATION PROBLEMS}

Problem 8. Non-invertible encryption procedure. The iteration of the chaotic systems sustaining chaos-based cryptosystems implies working with real numbers. Since the implementation of chaos-based cryptosystems is done with finite precision arithmetic, round-off operations could lead to a non-invertible encryption procedure.

One critical point when working with dynamical systems and the analysis of their dynamics is the selection of a right simulation framework. Indeed, the computer-based analysis of dynamical systems could lead to some conclusions different from those expected from theory. This divergence also influences and conditions chaos-based cryptosystems. Thus, if the characteristics and problems of finite-precision are not handled properly, then it is possible that the orbits generated as base of encryption procedure can not be regenerated exactly during the decryption stage and, consequently, the original plaintext can not be recovered even when the key is known. This problem is not only relevant for fixed-point arithmetic but also for floatingpoint one. Indeed, the round-off quantization errors could lead to the occurrence of a non-invertible function for encryption and, as a result, the decryption process will be impossible (see the cryptanalysis work in (Alvarez et al., 2007; Arroyo et al., 2008b,d; Solak and Çokal, 2008)).

Problem 9. Dynamical degradation. The implementation of chaotic systems in finite precision in digital computers leads often to dynamical properties completely different from the theoretical and expected ones. If this deviation is not considered during the design of chaosbased cryptosystems, it could imply a reduction of the performance and even a compromise of the security of the resulting cryptosystem.

This problem is closely related to the previous one, although the point of interest moves to degradation of dynamical properties of the implemented chaotic system with respect to the theoretical model. Consequently, the design of an encryption scheme using a chaotic system must be done by considering its practical implementation (not only the theoretical model). In (Alvarez and Li, 2006) some consequences of the dynamical degradation of a chaotic map are shown in the context of cryptography, whereas in (Li et al., 2005) one can find a thorough analysis of the dynamical degradation of a specific chaotic map and some ways to overcome this problem.

Problem 10. Lack of details in the description. According to Kerckhoffs' principle, the security of a cryptosystem can not be based on the secrecy of its encryption and decryption procedures. Furthermore, the key of any cryptosystem has to be easy to establish and to exchange, and the key space must be defined in an explicit and clear way.

The consecution of security through obscurity is something to avoid when designing an encryption scheme. All the operations involved in the encryption/decryption procedures must be verbosely explained, and the secret key must be clearly specified along with an exact estimation of the size of the key space. The security of the cryptosystem must be only related to the difficulty of guessing the key, and it can not depend on the lack of knowledge about the inner operating of the encryption and decryption procedures. Moreover, this lack of details implies a lack of security because without a careful investigation of the whole cryptography community many security holes might not be able to distinguished by the designers themselves. Refer to (Arroyo et al., 2008b) and (Li et al., 2008a) for a pair of examples.

\section{CONCLUSIONS}

As a result of all the cryptanalysis work in the field of chaos-based cryptography, we must conclude that the design of new strategies of encryption using chaos must be based on a good background on the theory of dynamical systems. In addition, cryptanalytic knowledge about previous proposals and the restrictions related to practical implementations on finite-precision machines must be carefully studied and handled. A cryptosystem is a chain composed of many links, whose security is determined by the weakest link, and cryptanalysis is the art of finding out the weakest link.

\section{REFERENCES}

Alvarez, G., Li, S., 2006. Breaking an encryption scheme based on chaotic baker map. Physics Letters A 352 (12), 78-82.

Alvarez, G., Li, S., Hernandez, L., 2007. Analysis of security problems in a medical image encryption system. Computers in Biology and Medicine 37 (3), 424-427.

Alvarez, G., Montoya, F., Pastor, G., 2003a. Cryptanalysis of a discrete chaotic cryptosystem using external key. Physics Letters A 319, 334-339.

Alvarez, G., Montoya, F., Romera, M., Pastor, G., 2003 b. Cryptanalysis of a chaotic secure communication system. Physics Letters A 306 (4), 200-205.

Alvarez, G., Montoya, F., Romera, M., Pastor, G., 2003c. Cryptanalysis of an ergodic chaotic cipher. Physics Letters A 311, 172-179.

Alvarez, G., Montoya, F., Romera, M., Pastor, G., 2004a. Cryptanalysis of dynamic look-up table based chaotic cryptosystems. Physics Letters A 326, 211-218. 
Alvarez, G., Montoya, F., Romera, M., Pastor, G., 2004b. Keystream cryptanalysis of a chaotic cryptographic method. Computer Physics Communications 156, 205207.

Arroyo, D., Alvarez, G., Li, S., Li, C., Fernandez, V., 2008a. Cryptanalysis of a new chaotic cryptosystem based on ergodicity. http://arxiv.org/abs/0806.3183.

Arroyo, D., Alvarez, G., Li, S., Li, C., Nunez, J., 2008b. Cryptanalysis of a discrete-time synchronous chaotic encryption system. Physics Letter A 372 (7), 1034-1039.

Arroyo, D., Li, C., Li, S., Alvarez, G., 2008c. Cryptanalysis of a computer cryptography scheme based on a filter bank. Chaos, Solitons and Fractals, In Press.

Arroyo, D., Rhouma, R., Alvarez, G., Li, S., Fernandez, V., 2008d. On the security of a new image encryption scheme based on chaotic map lattices. Chaos 18, Art. No. 033112, 7 pages.

Banerjee, S., Yorke, J. A., Grebogi, C., 1998. Robust chaos. Physical Review Letters 80, 14.

Chee, C. Y., Xu, D., 2006. Chaotic encryption using dicrete-time synchronous chaos. Physics Letters A 348 (3-6), 284-292.

García, P., Jiménez, J., 2002. Communication through chaotic map systems. Physics Letters A 298 (1), 35-40.

Kocarev, L., 2001. Chaos-based cryptography: A brief overview. IEEE Circuits and Systems Magazine 1 (2), 6-21.

Li, C., Li, S., Alvarez, G., Chen, G., Lo, K.-T., 2007. Cryptanalysis of two chaotic encryption schemes based on circular bit shift and XOR operations. Physics Letters A 369, 23-30.

Li, C., Li, S., Chen, G., Halang, W. A., 2008a. Cryptanalysis of an image encryption scheme based on a compound chaotic sequence. Image and Vision Computing. Article in Press.

Li, S., June 2003. Analyses and new designs of digital chaotic ciphers. Ph.D. thesis, School of Electronic and Information Engineering, Xi'an Jiaotong University, Xi'an, China, available online at http://www.hooklee.com/pub.html.

Li, S., Chen, G., Mou, X., 2005. On the dynamical degradation of digital piecewise linear chaotic maps. International Journal on Bifurcation and Chaos 15 (10), 31193151.

Li, S., Li, C., Chen, G., Bourbakis, N. G., Lo, K.-T., 2008 b. A general quantitative cryptanalysis of permutationonly multimedia ciphers against plaintext attacks. Signal Processing: Image Communication 23 (3), 212-223.

Pareek, N. K., Patidar, V., Sud, K. K., 2003. Discrete chaotic cryptography using external key. Physics Letters A 309, 75-82.

Pisarchik, A. N., Flores-Carmona, N. J., Carpio-Valadez, M., 2006. Encryption and decryption of images with chaotic map lattices. Chaos 16 (3), Art. No. 033118.

Skrobek, A., 2008. Approximation of a chaotic orbit as a cryptanalytical method on Baptista's cipher. Physics Letters A 372 (6), 849-859.

Solak, E., Çokal, C., 2008. Comment on "encryption and decryption of images with chaotic map lattices". Chaos 18 (3), 038101. 